\title{
AVALIAÇÃO DO EFEITO DO EXTRATO HIDROALCOÓLICO DE Schinus terebin- thifolius Raddi (AROEIRA) NO PROCESSO DE CICATRIZAÇÃO DA LINEA ALBA DE RATOS ${ }^{1}$
}

\author{
Evaluation of the hydro-alcoholic Schinus terebinthifolius raddi (Aroeira) extract in the heal- \\ ing process of the alba linea in rats
}

\begin{abstract}
José Aldemir Teixeira Nunes Jr. ${ }^{2}$, Jurandir Marcondes Ribas-Filho ${ }^{4}$, Osvaldo Malafaia ${ }^{4}$, Nicolau Gregori Czeczko ${ }^{4}$, Cristiano Machado Inácio ${ }^{5}$, Alexandre Wolanski Negrão ${ }^{5}$, Periguari Luiz Holanda de Lucena ${ }^{3}$, Hamilton Moreira ${ }^{4}$, Jorge Wagenfuhr Jr. ${ }^{5}$, Jordan de Jesus Cruz ${ }^{5}$
\end{abstract}

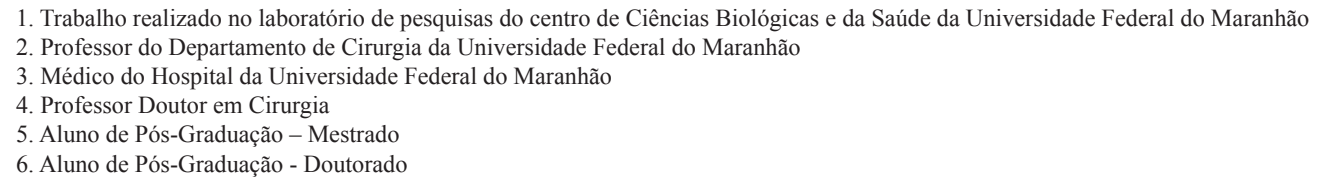

\section{RESUMO}

Objetivo: Avaliar o processo de cicatrização da parede abdominal de ratos após a injeção intraperitoneal do extrato hidroalcóolico de Schinus terebinthifolius Raddi. Métodos: Utilizaram-se 40 ratos da linhagem Wistar, distribuídos em dois grupos de 20 animais, cada um subdividido em dois subgrupos. Estes ratos foram mortos no $3^{\circ}$ e $7^{\circ}$ dias após a aplicação intraperitoneal do extrato. No grupo experimento, injetou-se uma única dose de extrato hidroalcoólico de Aroeira (100 mg por quilo de peso do animal) e no grupo controle uma única dose de solução salina isotônica a $0.9 \%$. Após a morte dos animais, realizou-se o inventário da cavidade peritoneal à procura de aderências, seguido da ressecção da parede abdominal anterior englobando a ferida operatória para análise. As aderências foram classificadas pelos critérios de adesão de Nair. Realizou-se avaliação tensiométrica da parede abdominal através da medida da carga máxima suportada e da deformação máxima, medidos em máquina universal de ensaios do tipo Tiratest. Por fim, a avaliação histológica foi realizada através da coloração hematoxilina-eosina, com análise dos parâmetros: inflamação aguda, inflamação crônica, necrose isquêmica, reação gigantocelular do tipo corpo estranho, proliferação fibroblástica, fibrose, reepitelização e coaptação das bordas da sutura. Resultados: A avaliacão macroscópica não revelou presença de aderências significativas entre a linha alba e as vísceras intra-abdominais nos grupos de estudo. A tensiometria demonstrou aumento significativo das variáveis carga máxima e deformação máxima ( $\mathrm{p}=0,006$ e $\mathrm{p}=0,000$ respectivamente) entre os grupos controle e experimento de sete dias. A avaliação histológica intergrupos (controle e experimento) de três e sete dias não demonstrou alteração significativa para os parâmetros neoformação vascular, necrose, fibrose, reepitelização e coaptação das bordas da sutura. Notou-se diferença significativa para proliferação fibroblástica $(\mathrm{p}=0,014)$ na avaliação intergrupo de três dias, e para inflamação crônica $(p=0,023)$ e reação gigantocelular do tipo corpo estranho $(p=0,008)$ na avaliação intergrupo de sete dias. Na análise intragrupo controle, houve diferença significativa para inflamação crônica no subgrupo três dias, e, finalmente, na análise intragrupo experimento, observou-se diferença significativa para inflamação aguda e proliferação de fibroblastos $(p=0,001$ e $p=0,020)$ no subgrupo de três dias em relação ao subgrupo de sete. Conclusão: A injeção intraperitoneal do extrato hidroalcoólico de Schinus terebinthifolius Raddi em laparotomias medianas de ratos não alterou a cicatrização na análise macroscópica e induziu a aumento da carga máxima de ruptura e deformação máxima da linha alba na análise tensiométrica. Na análise histológica, determinou efeito cicatrizante no subgrupo de animais experimento de três dias. Descritores: Cicatrização de Feridas. Ratos. Aroeira Brasileira. Linea Alba

\footnotetext{
ABSTRACT

Purpose: To evaluate the healing process in the abdominal wall of rats after the intra-peritoneal injection of the hydroalcoholic Schinus terebinthifolius Raddi extract. Methods: Forty Wistar rats were used, distributed in two groups of 20 animals, divided into two subgroups, according to the death day, three or seven, after the intra-peritoneal injection of the extract. The experimental group was injected with only one dose of the hydro-alcoholic Aroeira extract (100mg per animal kilogram); the control group was injected with only one dose of isotonic saline solution at $0.9 \%$. After the animal death, an inventory of the peritoneal cavity was carried out in a careful search for any adhesion, followed by the resec-
} 
tion of the anterior abdominal wall encompassing the operating incision in order to examine it for evidence of infection. The adhesions were classified according to the Nair criteria. The tensiometric assessment was performed by means of the measurement of the maximum charge and maximum deformity tolerated, measured in a universal Tiratest type testing machine and, finally, the histological evaluation was performed by means of the hematoxylin-eosine staining in following parameters: severe inflammation, chronic inflammation, ischemic necrosis, gigantocellular reaction of the foreign body type, fibroblastic proliferation, fibrosis, re-epithelization and co-optation of the suture borders. Results: The macroscopic evaluation didn't have any evidence of significant adherence between the alba linea and the intra-abdominal viscera in the study groups. The tensiometry showed significant effect for the Maximum Charge and Maximum Deformity ( $\mathrm{p}=0.006$ and $\mathrm{p}=0.000$, respectively) between the 7-day control and experimental groups. The histological 3-day and 7-day control and experimental intergroup evaluation did not show significant alteration for the parameters vascular neoformation, necrosis, fibrosis, re-epithelization and co-aptation. A significant difference was perceived for fibroblastic proliferation $(\mathrm{p}=0.014)$ in the 3-day intergroup evaluation and for chronic inflammation $(\mathrm{p}=0.023)$ and gigantocellular reaction of the foreign body type $(\mathrm{p}=0.008)$ in the 7-day intergroup evaluation. The control intragroup analysis, had a significant difference for chronic inflammation in the 3-day group and, finally, in the experimental intragroup analysis, a significant difference was observed for acute inflammation and fibroblastic proliferation $(\mathrm{p}=0.001$ and $\mathrm{p}=0.020)$ in the 3-day regarding the 7-day group. Conclusion: The intraperitoneal injection of the Schinus terebinthifolius Raddi hydro-alcoholic extract in median laparotomies in rats has not determined any significant alteration in the macroscopic analysis, but it induced an increase of maximum charge of rupture and maximum deformity of the alba linea in the tensiometric analysis. In the histological point of view, the Aroeira group had a positive healing effect on the 3-day.

Key Words: Wound Healing. Rats. Peppertree Brazilian. Alba Linea

\section{Introdução}

O tratamento das feridas, objetivando a melhora do processo de cicatrização com resultado estético e funcional satisfatórios, desafiou os cirurgiões a modificar suas atitudes ao longo dos séculos ${ }^{1}$.

O processo de cicatrização é evento biológico complexo, envolvendo inflamação, quimiotaxia, proliferação celular, diferenciação e remodelação. A cicatrização é essencial para a manutenção da integridade do organismo, entretanto é processo que não está completamente esclarecido, principalmente em relação ao aspecto do uso de substâncias químicas que possam agilizá-lo ou retardá-1o ${ }^{2,3}$.

Encontram-se relatos de várias substâncias que foram testadas e usadas no processo de cicatrização de feridas. $\mathrm{O}$ uso tópico da papaína apresentou resultados satisfatórios em pacientes com lesões de diversas origens ${ }^{4}$. O óleo da rosa-mosqueta mostrou-se eficaz no tratamento de feridas cutâneas abertas em ratos, favorecendo a cicatrização ${ }^{5}$. O extrato alcoólico de flores da Ixora coccinea (ixora) facilitou a cicatrização com aumento da granulação, da força de tensão e da deposição de colágeno em feridas cutâneas abertas de ratos $^{6}$. A Bryophyllum pinnata (Siempreviva) mostrou-se eficaz no tratamento de feridas cutâneas abertas em ratos; porém, existem poucos trabalhos demonstrando o efeito das plantas medicinais nesta área de pesquisa ${ }^{7}$.

AAroeira (Schinus terebinthifolius Raddi) pertencente à família das anacardeáceas é uma planta comum da vegetação litorânea dos estados nordestinos brasileiros desde o Rio Grande do Norte até Sergipe. A sua casca é indicada como anti-inflamatório e cicatrizante ${ }^{8}$.

O objetivo do presente estudo é avaliar o efeito do extrato hidroalcóolico da entrecasca da Aroeira injetado por via intraperitoneal no processo de cicatrização tecidual da parede abdominal de ratos, através da análise macroscópica, tensiométrica e microscópica.

\section{Métodos}

Este trabalho foi realizado no laboratório de pesquisa do Departamento de Fisiologia e Farmacologia do Centro de Ciências Biológicas e da Saúde da Universidade Federal do Maranhão (UFMA) e foi aprovado pelo Comitê de Ética em Pesquisa da UFMA, São Luís, MA - Brasil.

\section{Amostra, identificação dos animais e divisão dos grupos}

Foram estudados 40 ratos (Rattus norvegicus albinus) machos, da linhagem Wistar, pesando entre 100 e $150 \mathrm{~g}$. Os animais foram divididos de maneira aleatória e distribuídos em dois grupos de 20 (grupos controle e experimento). Cada um desses grupos foi dividido em dois subgrupos de 10 ratos, de acordo com a data da sua morte: grupo controle de três dias (GC3D), grupo controle de sete dias (GC7D), grupo experimento de três dias (GE3D) e grupo experimento de sete dias (GE7D).

\section{Preparação do extrato da Aroeira}

As amostras da entrecasca da Aroeira foram obtidas de um exemplar adulto da planta na fazenda Santana, no município de Palmeirantes, no Estado do Tocantins. O preparo do extrato hidroalcóolico da Aroeira foi realizado conforme a metodologia preconizada anteriormente ${ }^{7}$. O extrato está identificado e catalogado no Herbário Seabra do Departamento de Farmácia do Centro de Ciências Biológicas e da Saúde da UFMA sob o número 488.

\section{Etapas experimentais}

Os animais foram pesados e submetidos à anestesia inalatória dentro de uma campânula com algodão embebido com éter sulfúrico comercial a 97\%, em sistema fechado, com tempo médio de cinco minutos. Foram considerados 
anestesiados quando apresentavam-se imóveis e com perda do reflexo corneano. A anestesia foi mantida em sistema semi-aberto sob máscara artesanal. Os animais, após pesados e identificados com ácido pícrico, foram imobilizados em decúbito dorsal. Realizou-se a epilação da região tóracoabdominal em uma extensão de 4,0 x 3,0 cm .

A antissepsia do terço inferior do tórax e do abdômen foi realizada com PVPI (Povidine ${ }^{\circledR}$, Darrow ${ }^{\circledR}$ ). Realizou-se uma incisão longitudinal mediana iniciando-se logo abaixo do processo xifóide e estendendo-se inferiormente por 3,0 $\mathrm{cm}$, compreendendo a pele e o subcutâneo, identificando-se a linea alba.

A seguir, realizou-se a abertura da linha alba e do peritôneo, expondo-se a cavidade peritoneal. Após a exposição da cavidade peritoneal e sem qualquer manuseio das vísceras, injetou-se soro fisiológico $(1 \mathrm{ml} / \mathrm{kg})$ à temperatura ambiente (GC) ou extrato de Schinus terebinthifolius Raddi na dose de $100 \mathrm{mg} / \mathrm{kg}$ de peso do animal (GE).

A síntese da parede abdominal foi feita em plano único, com seis pontos separados, com fio monofilamentar de polipropileno 5-0 (Prolene ${ }^{\circledR}$, Ethicon ${ }^{\circledR}$ ).

No pós-operatório os animais foram examinados diariamente, anotando-se o peso, as condições comportamentais e o aspecto da ferida operatória com os dados anotados em protocolo próprio.

Os animais de cada grupo quando de seu sacrifício foram pesados e colocados sob campânula de vidro e submetidos a dose letal inalatória de éter sulfúrico a $97 \%$, sendo retirados após a ausência completa de reflexos e de movimentos respiratórios.

\section{Avaliação macroscópica}

Após a morte, os animais foram fixados à mesa cirúrgica, realizando-se a dissecção da pele e tecido subcutâneo, expondo-se a musculatura abdominal anterior e a cicatriz cirúrgica, e, em seguida, foram realizadas duas incisões transversas no abdômen: uma inferior ao processo xifóide e outra na pelve $1 \mathrm{~cm}$ abaixo da borda inferior da ferida operatória. A seguir, realizou-se uma incisão longitudinal na porção mais lateral da parede abdominal à direita da linha média, entre as duas incisões transversas, seccionando-se completamente uma das bordas e, deslocando-a contralateralmente, realizou-se a exposição completa seguida da inspeção da cavidade peritoneal. Realizou-se a ressecção de toda a parede anterior do abdômen, compreendendo a incisão operatória e os tecidos circunjacentes com dimensões de 5 $\mathrm{cm}$ no sentido crânio-caudal e $3 \mathrm{~cm}$ no látero-lateral. A peça cirúrgica ressecada foi colocada sobre o campo cirúrgico e examinada para verificação de abscessos e deiscência da sutura. Em seguida foram depositadas em frascos, que foram identificados de acordo com o grupo, o subgrupo e a numeração do animal. O inventário da cavidade foi realizado para detectar deiscências, abscessos e aderências. Utilizou-se o escore de adesão de Nair ${ }^{9}$, para a quantificação das aderências.

\section{Avaliação tensiométrica}

O estudo tensiométrico da parede abdominal foi realizado no Laboratório de Ensaios Mecânicos do Centro Federal Tecnológico do Maranhão (CEFET-MA), São Luis - MA, Brasil em máquina universal de ensaios - modelo Tiratest 2420, TIRA Maschinenbau Gmbh.

Após a retirada de fragmento para a histologia, o restante da peça cirúrgica medindo $3,5 \mathrm{~cm}$ crânio-caudal e $2,0 \mathrm{~cm}$ látero-lateral (Figura 1) foi colocada em um frasco contendo solução salina isotônica a $0,9 \%$ e encaminhada para a realização do teste de tração.

Imediatamente, antes do ensaio mecânico, o comprimento da peça cirúrgica foi medido com um paquímetro de grande precisão (Mitutoyo ${ }^{\circledR}$ ) e pesada em balança digital com precisão de 0,01 grama. Inicialmente, após ter sido fixado na máquina universal de ensaios, a peça recebeu uma pré-carga de $2 \mathrm{~N}$, com tempo de acomodação de 60 segundos. A velocidade estabelecida para todos os ensaios

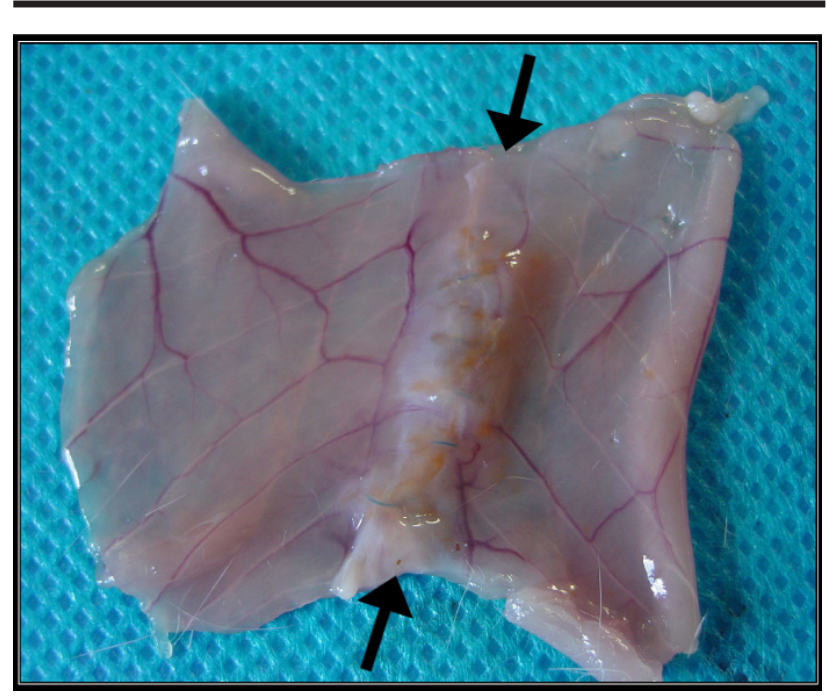

FIGURA 1 - Parede abdominal anterior ressecada. no $7^{\circ}$ dia de P.O. Nota: Setas $=$ ferida cirúrgica e sutura.

foi de $5 \mathrm{~mm} /$ minuto e as medidas foram realizadas a cada $0,5 \mathrm{~mm}$

Os testes de tração (Figura 2) originaram os gráficos de carga versus deformação, obtidos de cada ensaio, a partir dos quais foram calculadas as propriedades mecânicas e o limite máximo, que é o maior valor da carga máxima de ruptura (Cmáx) e a deformação máxima de ruptura (Dmáx). A primeira está relacionada com a resistência mecânica e, no caso da linea alba, com o conteúdo de fibras colágenas, enquanto que a segunda está relacionada com a elasticidade e, neste caso, com o conteúdo de fibras elásticas.

Após a fixação das amostras nos acessórios da máquina universal de ensaios, elas foram submetidas à tração axial. Os ensaios foram realizados até ser atingido o limite máximo e, após isto, continuou-se a registrar as cargas correspondentes a cada deformação medida. Ao final, foi realizada inspeção do local de ruptura da peça 


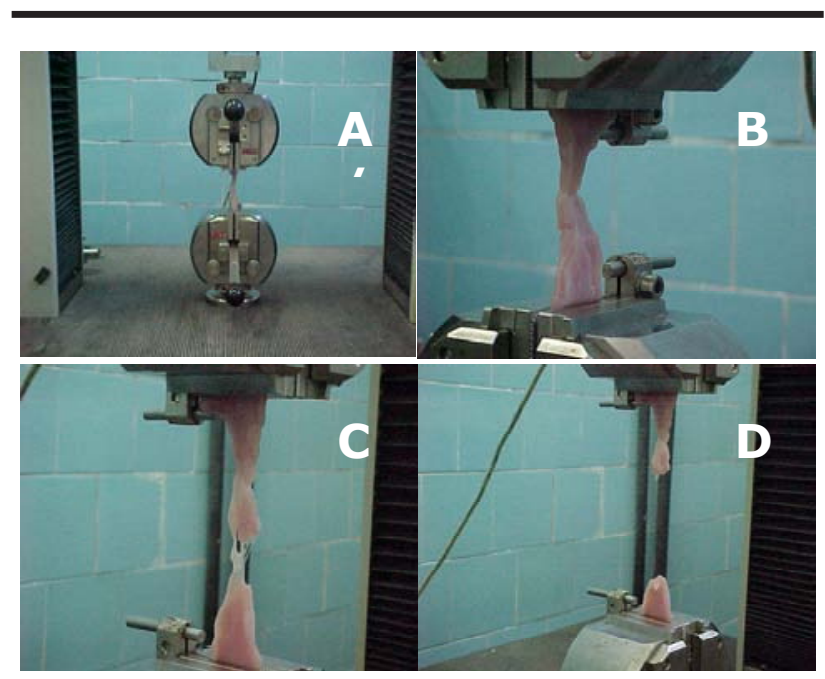

FIGURA 2 - Teste de tração. Legenda: A- Momento de posicionamento, sem tração. B- Momento de tração, ainda sem ruptura. C-Momento inicial da ruptura. D- Momento final de separação total dos cotos da peça.

\section{Avaliação microscópica}

Retirou-se uma faixa horizontal de $1 \mathrm{~cm}$, englobando parte da ferida operatória, para ser encaminhado ao laboratório de histologia da UFMA, onde os processos de rotina foram realizados através da coloração de hematoxilina-eosina.

\section{Análise estatística}

Os dados foram analisados utilizando-se o programa Statistica for Windows 5.1. As variáveis tensiométricas Deformação Máxima (DMáx) e Carga Máxima (CMáx) foram avaliadas pelo teste $t$ de Student, sendo que para se avaliar conjuntamente os efeitos do grupo (GC e GE), do tempo (três e sete dias) e a interação entre eles fez-se uma ANOVA com dois fatores. Quando ocorria efeito significativo de um dos fatores, fazia-se o teste de Tuckey e compararam-se as médias. As variáveis da avaliação macroscópica e microscópica foram analisadas pelo teste não-paramétrico de Mann-Whitney. O nível de significância utilizado para se rejeitar a hipótese de nulidade foi de 0,05 .

\section{Resultados}

Os animais apresentaram pós-operatório sem complicações, permanecendo vivos até o final do experimento.

\section{Avaliação macroscópica}

A avaliação da superfície externa da ferida operatória não demonstrou a presença de abscesso ou deiscência em nenhum dos animais.

Observou-se a presença de aderências frouxas entre alças intestinais e a parede abdominal (escore I de Nair) em dois animais do GC3D (20\%), em dois do GC7D (20\%), em um do GE3D (10\%) e em três no GE7D (30\%).
A análise estatística entre GC e GE aos três e sete dias ( $p$ $=0.653$ ) e aquela feita entre todos os grupos controle e todos grupos experimentos individualizados entre si (intergrupos), de três e sete dias $(\mathrm{p}=0,705)$ não demonstrou diferença estatisticamente significante. A análise estatística intragrupos controle de três e sete dias $(\mathrm{p}=0.935)$ e a análise intragrupos experimento de três e sete dias $(\mathrm{p}=0.450)$ demonstrou-se sem diferença estatisticamente significante.

\section{Avaliação tensiométrica}

O estudo tensiométrico foi feito na totalidade das peças cirúrgicas e todas romperam no local da sutura.

Os valores das propriedades mecânicas obtidas para os GC e GE, após três e sete dias de operados, são apresentados nas Tabelas 1, 23 e 4, com as suas respectivas médias e desvio-padrão.

A análise estatística intergrupos de três dias para as

TABELA 1 - Valores das propriedades mecânicas dos animais do grupo controle três dias após a operação

\begin{tabular}{lcc}
\hline Rato & Carga Máxima & $\begin{array}{c}\text { Deformação } \\
\text { Máxima }\end{array}$ \\
\hline Rato 1 & 3.38 & 22.77 \\
Rato 2 & 3.72 & 18.10 \\
Rato 3 & 7.19 & 25.57 \\
Rato 4 & 3.66 & 18.18 \\
Rato 5 & 5.01 & 19.40 \\
Rato 6 & 4.30 & 31.42 \\
Rato 7 & 7.12 & 33.78 \\
Rato 8 & 3.95 & 26.81 \\
Rato 9 & 7.68 & 27.74 \\
Rato 10 & 3.12 & 24.45 \\
\hline Média & $\mathbf{4 . 9 1}$ & $\mathbf{2 4 . 8 2}$ \\
\hline Desvio Padrão & $\mathbf{1 . 7 5}$ & $\mathbf{5 . 3 7}$ \\
\hline
\end{tabular}

TABELA 2 - Valores das propriedades mecânicas dos animais do grupo experimento três dias após a operação

\begin{tabular}{lcc}
\hline Rato & Carga Máxima & $\begin{array}{c}\text { Deformação } \\
\text { Máxima }\end{array}$ \\
\hline Rato 1 & 2.95 & 23.27 \\
Rato 2 & 3.24 & 19.80 \\
Rato 3 & 6.59 & 30.35 \\
Rato 4 & 6.44 & 28.96 \\
Rato 5 & 2.84 & 24.09 \\
Rato 6 & 6.42 & 24.38 \\
Rato 7 & 11.34 & 25.92 \\
Rato 8 & 5.13 & 23.12 \\
Rato 9 & 7.74 & 23.76 \\
Rato 10 & 2.61 & 23.78 \\
\hline Média & $\mathbf{5 . 5 3}$ & $\mathbf{2 4 . 7 4}$ \\
\hline Desvio Padrão & $\mathbf{2 . 7 7}$ & $\mathbf{3 . 0 2}$ \\
\hline
\end{tabular}

variáveis Cmáx e Dmáx, cujos valores das propriedades mecânicas estão contidos nas Tabelas 1 e 2; demonstra $\mathrm{p}=0,559$ e $\mathrm{p}=0,968$ respectivamente, não havendo, portanto, 
diferença estatisticamente significante.

A análise nos grupos de sete dias, cujos valores das propriedades mecânicas estão expostos nas Tabelas 3 e 4; revela $\mathrm{p}=0,006$ para Cmáx e $\mathrm{p}=0,000$ para Dmáx, havendo diferença estatisticamente significativa entre os grupos de animais estudados.

TABELA 3 - Valores das propriedades mecânicas dos animais do grupo controle sete dias após a operação

\begin{tabular}{lcc}
\hline Rato & Carga Máxima & $\begin{array}{c}\text { Deformação } \\
\text { Máxima }\end{array}$ \\
\hline Rato 1 & 5.93 & 23.62 \\
Rato 2 & 6.56 & 22.05 \\
Rato 3 & 4.95 & 23.62 \\
Rato 4 & 6.53 & 27.53 \\
Rato 5 & 7.97 & 21.08 \\
Rato 6 & 3.34 & 27.24 \\
Rato 7 & 3.92 & 19.67 \\
Rato 8 & 4.58 & 21.42 \\
Rato 9 & 3.37 & 32.31 \\
Rato 10 & 3.27 & 38.73 \\
\hline Média & $\mathbf{5 . 0 4}$ & $\mathbf{2 5 . 7 2}$ \\
\hline Desvio Padrão & $\mathbf{1 . 6 4}$ & $\mathbf{5 . 7 0}$ \\
\hline
\end{tabular}

TABELA 4 - Valores das propriedades mecânicas dos animais do grupo experimento sete dias após a operação

\begin{tabular}{lcc}
\hline Rato & Carga Máxima & $\begin{array}{c}\text { Deformação } \\
\text { Máxima }\end{array}$ \\
\hline Rato 1 & 7.38 & 31.22 \\
Rato 2 & 9.16 & 43.62 \\
Rato 3 & 7.40 & 33.85 \\
Rato 4 & 6.54 & 39.21 \\
Rato 5 & 7.25 & 32.35 \\
Rato 6 & 8.31 & 30.19 \\
Rato 7 & 6.29 & 42.82 \\
Rato 8 & 5.45 & 33.98 \\
Rato 9 & 5.35 & 38.90 \\
Rato 10 & 7.29 & 33.30 \\
\hline Média & $\mathbf{7 . 0 4}$ & $\mathbf{3 5 . 9 4}$ \\
\hline Desvio Padrão & $\mathbf{1 . 1 9}$ & $\mathbf{4 . 8 2}$ \\
\hline
\end{tabular}

Avaliação microscópica

A Tabela 5 mostra os valores da densidade celular no estudo histológico para o $3^{\circ}$ dia. A análise estatística demonstrou aumento da proliferação fibroblástica $(\mathrm{p}=0,014)$ no $\mathrm{GE}$, ocorrendo diferença estatisticamente significativa entre os dois grupos.

A Figura 3 mostra os achados de um animal do GE3D com proliferação fibroblástica e colagenização moderada.

A Tabela 6 mostra os valores da densidade celular no estudo histológico para o $7^{\circ}$ dia e demonstrou um efeito significativo para a variável inflamação crônica com p=0,023.

A Figura 4 demonstra a intensidade do processo inflamatório crônico.

Observou-se também (Tabela 6) diferença significativa com aumento da reação gigantocelular no GE de sete dias $(\mathrm{p}=0,008)$.
TABELA 5 - Análise dos grupos controle e experimento três dias após a operação

\begin{tabular}{lccc}
\hline Variáveis histológicas & SGC3D & SGE3D & P \\
\hline Inflamação aguda & 69,5 & 120,5 & 0,090 \\
Inflamação crônica & 83,5 & 106,5 & 0,596 \\
Necrose & 90,0 & 100 & 1,000 \\
Reação gigantocelular & 90,0 & 100 & 1,000 \\
Proliferação fibroblástica & 60,0 & 130 & $* 0,014$ \\
Colagenização & 84,0 & 106 & 0,624 \\
Reepitelização & 90,0 & 100 & 1,000 \\
Coaptação das bordas & 90,0 & 100 & 1,000 \\
Extensão do proces s o & 84,5 & 105,5 & 0,653 \\
inflamatório da parede & & 122,5 & 0,066 \\
Neoformação vascular & 67,5 & \multicolumn{3}{c}{} \\
\hline
\end{tabular}

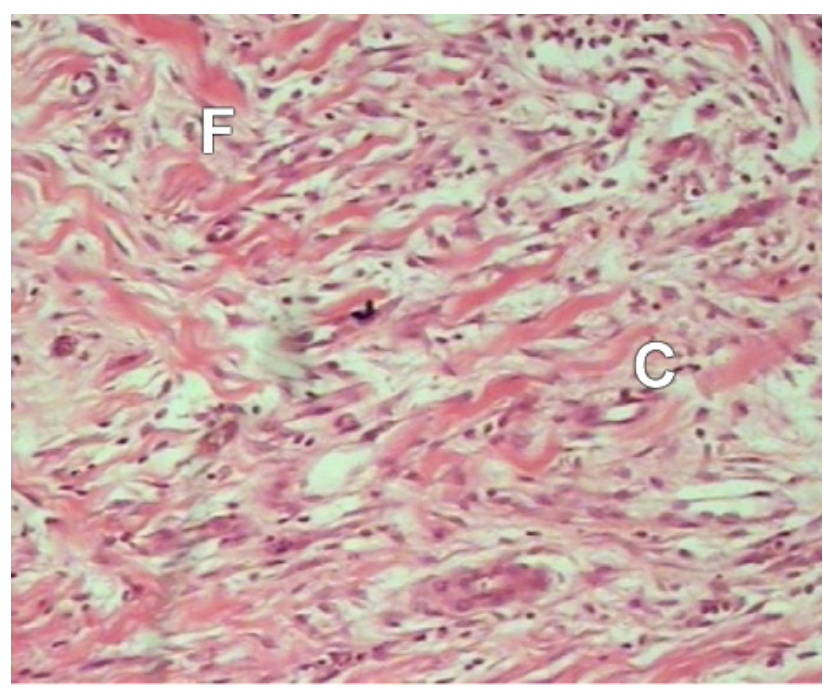

FIGURA 3 - Fotomicrografia mostrando proliferação fibroblástica e colagenização no $3^{\circ}$ dia de P.O. animal do grupo GE3D (Coloração HE, aumento de 100x) mostrando proliferação fibroblástica. Nota: $\mathrm{C}=$ colágeno, $\mathrm{F}=$ fibroblasto.

TABELA 6 - Análise dos grupos controle e experimento três dias após a operação

\begin{tabular}{lccc}
\hline Variáveis histológicas & SGC7 & SGE7 & P \\
\hline Inflamação aguda & 110 & 100 & 0,705 \\
Inflamação crônica & 75 & 135 & $* 0,023$ \\
Necrose & 105 & 105 & 1,000 \\
Reação gigantocelular & 70 & 140 & $* 0,008$ \\
Proliferação fibroblástica & 90,5 & 99,5 & 0,438 \\
Colagenização & 85 & 125 & 0,131 \\
Reepitelização & 100 & 110 & 0,705 \\
Coaptação das bordas & 105 & 105 & 1,000 \\
Extensão do proces so & 85 & 105 & 0,683 \\
inflamatório da parede & & & \\
Neoformação vascular & 84 & 126 & 0,112 \\
\hline
\end{tabular}




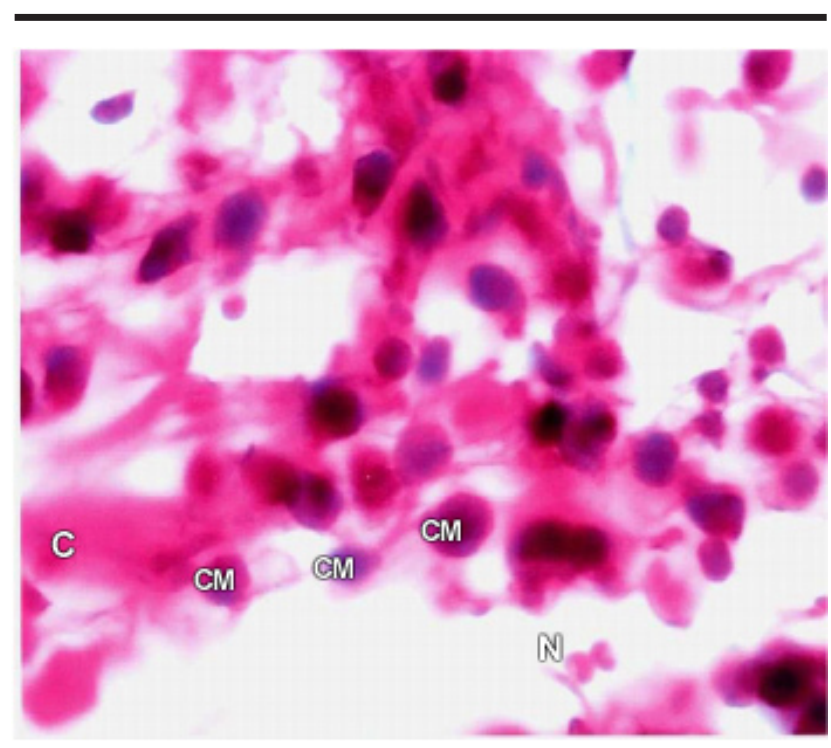

FIGURA 4 - Fotomicrografia mostrando a intensidade da reação inflamatória crônica no $7^{\circ}$ dia de P.O. animal do grupo GE7D (coloração HE, aumento de 100x). Nota: $\mathrm{C}=$ colágeno. $\mathrm{CM}=$ Células mononucleares. $\mathrm{N}=$ Neutrófilos

Na análise intragrupo controle, notou-se diferença significativa para inflamação crônica no grupo três dias e na do experimento, diferença significativa para inflamação aguda $(\mathrm{p}=0,001)$, reação gigantocelular $(\mathrm{p}=0,008)$ e proliferação de fibroblastos $(\mathrm{p}=0,020)$ no grupo de três dias.

\section{Discussão}

Diferentemente dos achados de Araújo ${ }^{10}$ que observou alto número de aderências na laparotomia em ratos, notouse neste estudo apenas aderências entre a linha alba e as vísceras abdominais em poucos animais, sendo que não houve diferença significante entre os grupos controle e experimento, empregando-se a avaliação pelo escore de Nair ${ }^{9}$. À semelhança do trabalho de Mazzini et al. ${ }^{11}$, observou-se baixo número de aderências, sendo que a maioria delas eram frouxas.

Outros autores estudaram a utilização de substâncias injetadas na cavidade peritoneal com o objetivo de diminuir a formação de aderências. Demonstraram que o uso de parafina líquida ${ }^{12}$ e de fosfolipídeos ${ }^{13}$ reduzem a formação de aderências intraperitoneais sem aumentarem o número de complicações sépticas. Ao contrário destes autores, não observou-se neste trabalho diferença significativa entre os grupos estudados a ponto de ser justificada a aplicação de qualquer substância para diminuir as aderências intracavitárias $^{14,15}$.

O uso da tensiometria na cicatrização da linha alba tem sido descrito por vários autores e foi padronizado por Tognini et al. ${ }^{16}$ no seu estudo de fechamento da parede abdominal de ratos com pontos contínuos e separados. Utilizou uma máquina universal de ensaios, com a velocidade de 30 milímetros por minuto. Nesta pesquisa, utilizaram-se equipamento e técnica semelhantes, mas a velocidade do ensaio foi diferente para menor, afim de minimizar o risco de deformação e ruptura das extremidades das peças presas às garras do braço do equipamento de tração, evitando-se assim perda de amostras ${ }^{17}$.

Em comparação com os estudos de Araújo ${ }^{10}$ e de Mazzini et al. ${ }^{11}$, observou-se similaridade nos resultados dos testes tensiométricos, havendo aumento da Cmáx e também da Dmáx, embora o autor não tenha empregado telas no fechamento da parede abdominal, inferindo um efeito cicatrizante do extrato de Schinus terebinthifolius Raddi.

À semelhança do estudo de Ogata $^{18}$, os testes de tração demonstraram que houve aumento da resistência das suturas, o que pode ser comparável com o grupo de animais operados mais tardiamente, em que a Cmáx e a Dmáx suportadas pelas peças foram significativamente maiores que o grupo controle.

Conforme já descrito na literatura ${ }^{8}$, o elemento essencial do fibroblasto, o colágeno, proporciona a força de estiramento das feridas em cicatrização e a sua quantidade e estabilidade refletem-se na resistência da ferida, e, portanto, na sua carga de ruptura. Neste estudo, ficou demonstrado aumento da quantidade de fibroblastos no $3^{\circ}$ e no $7^{\circ}$ dias no grupo de animais que utilizou o extrato e isto se refletiu em aumento da Cmáx de ruptura, com análise estatística demonstrando diferença significativa para este grupo.

Com relação ao alongamento da ruptura (Dmáx), o qual reflete o conteúdo de fibras elásticas, houve diferenças estatísticas entre os dois grupos, diferente dos estudos de Seckel, Younai e Wang (1998) citados por Mesquita ${ }^{19}$ que observaram perda do conteúdo de fibras elásticas e ausência de diferenças significativas nos grupos estudados.

A proliferação fibroblástica e a deposição de colágeno são responsáveis pelo desenvolvimento de força tênsil adequada para uma boa evolução da cicatriz. A partir do $3^{\circ}$ dia do início do processo de cicatrização já se encontram fibroblastos e colágeno na área da sutura. Entre o $7^{\circ}$ e o $14^{\circ}$ dias a síntese do colágeno atinge seu ápice ${ }^{19}$. No ensaio aqui apresentado evidenciou-se que o extrato de Aroeira tem ação cicatrizante, uma vez que houve aumento dos fibroblastos na região da sutura no grupo de animais do $3^{\circ}$ e $7^{\circ}$ dias.

A coloração pela HE foi escolhida por se tratar de corante universal, usado rotineiramente para avaliações em estudos histológicos. É método de coloração simples e barato, adequado para quantificar e identificar os elementos celulares envolvidos no processo cicatricial. Outros autores ${ }^{7,19,20}$ utilizando-se de colorações tipo Picrosirius red e tricrômio de Masson, obtiveram resultados semelhantes à HE. O corante utilizado na hematoxilina-eosina revelou-se adequado, não havendo necessidade do uso de colorações adicionais.

A presença de processo inflamatório crônico, com aumento de fibroblastos e reações gigantocelulares, promoveu melhora na cicatrização, o que se refletiu no aumento da resistência das suturas no $7^{\circ}$ dia. Apesar deste período ainda não ser o ideal para a análise devido ao colágeno ainda estar imaturo $^{8,15}$, houve diferença significativa entre os dois grupos estudados.

\section{Conclusão}

A injeção intraperitoneal do extrato hidroalcoólico de 
Schinus terebinthifolius Raddi em laparotomias medianas de ratos não alterou a cicatrização na análise macroscópica, mas induziu aumento da carga máxima de ruptura e deformação máxima da linea alba na análise tensiométrica. $\mathrm{Na}$ análise histológica evidenciou efeito cicatrizante no grupo experimento de três dias.

\section{Referências}

1. Modolin M, Bevilacqua, RG. Cicatrização de feridas: síntese das aquisições recentes. Rev Bras Clin Ter. 1985;14:208-13.

2. Araújo GF. Implante de tela de algodão na parede abdominal de ratos.[Tese -Doutorado]. São Paulo: Universidade Federal de São Paulo, Escola Paulista de Medicina; 1997.

3. Sanchez-Neto R, Barone B, Teves DC, Simões M, Juliano Y. Aspectos morfológicos e morfométricos da reparação tecidual de feridas cutâneas de ratos com e sem tratamento com solução de papaína a 2\%. Acta Cir Bras. 1993;8:18-23.

4. Masini E, Calamo MA. Uma forma de tratamento de lesões cutâneas com papaína e sacarose. Rev Bras Clin Ter. 1986;15:208-13.

5. Marchini FB, Martins DMFS, Teves DC, Simões MJ. Efeito do óleo de rosa mosqueta na cicatrização de feridas abertas. Rev Paul Med. 1988;106(6):356.

6. Navay BS, Udupa AL, Udupa SL. Effect of Ixora coccinea flowers on dead space wound healing in rats. Fitoter. 1999;70:233-6.

7. Oliveira AB. Espectrofotometria no controle de qualidade de fitoterápicos da Schinus terebinthifolius Raddi: uso de marcadores. [Dissertação-Mestrado]. São Luis: Universidade Federal do Maranhão; 2001.

8. Carmello-Guerreiro SM, Paoli AAS. Morfologia e anatomia da semente de Schinus terebinthifoliusRaddi (anacardiaceae) em desenvolvimento. Rev Bras Bot.1999; 22:1-12.

9. Nair SK, Bhat IK, Aurora AL. Role of proteolytic enzymes in prevention of postoperative intraperitoneal adhesions. Arch. Surg 1974; 108: 849-53.
10. Araújo GF. Implante de tela de algodão na parede abdominal de ratos. [Tese-Doutorado]. São Paulo: Universidade Federal de São Paulo, Escola Paulista de Medicina; 1997.

11. Mazzini DL, Mantovani M. Fechamento da parede abdominal com afastamento parcial das bordas da aponeurose utilizando sobreposição com telas de vicryil ou marlex em ratos. Acta Cir Bras. 1999; 14(1):28-34.

12. Ahuja M, Singh VP, Kumar A. Prevention of postoperative intraperitoneal adhesions an experimental study in rats. UC Chakraborti award session at the 27th Annual conference of IAPS, 2001.

13. Muller AS, Treutner KH, Haase G, Kinzel S, Tietze L, Schumpelick V. Effect of intraperitoneal antiadhesive fluids in a rat peritonitis model. Arch Surg. 2003;138:286-90.

14. Gabbiani G, Majno G. Dupuytren's contracture: fibroblast contraction? A ultrastructural study. Am J Pathol. 1972; 66: 131-8.

15. White M N, Johnson AC, Eger KL. Anesthesy in experimental surgery. Exp Surg. 1974; 1: 250-60.

16. Tognini JRF, Goldenberg S, Naresse LE, Simões M, Alves FLG, Magalhães AM. Estudo comparativo entre a sutura contínua e a com pontos separados na parede abdominal de ratos. Acta Cir Bras. 1997;12(4):249-54.

17. Rêgo TJAS. Fitogeografia das plantas medicinais do Maranhão. In: Levantamento de plantas medicinais da região dos chapadões maranhenses.São Luís: EDUFMA/ CORSUP, 1993. p. 27-43

18. Ogata AC. Prótese externa de politetrafluoroetileno como reforço de anastomose traqueal em coelhos - avaliação da resistência à tensão. [Disseratação - Mestrado]. Curitiba: Universidade Federal do Paraná; 2002.

19. Mesquita LAF. Estudo da cicatrização cutânea causada pelo laser de $\mathrm{CO} 2$ ultrapulsado em retalho dorsal de ratos. [Dissertação-Mestrado]. Curitiba: Instituto de Pesquisas Médicas, Hospital Universitário Evangélico de Curitiba; 2002.

20. Witte MB, Barbul A. Princípios gerais da cicatrização das feridas. Clin Cir Am North. 1997; 77:509-27. 
Recebimento: 22/01/2005

Revisão: 23/05/2005

Aprovação: 17/06/2006

\section{Como citar este artigo:}

Nunes Jr JAT, Ribas-Filho JM, Malafaia O, Czeczko NG, Inácio CM, Negrão AW, Lecuna PLH, Moreira H, Wagenfuhr Jr J, Cruz JJ. Avaliação do efeito do extrato hidroalcoólico de Schinus terebinthifolius Raddi (Aroeira) no processo de cicatrização da linea alba de ratos. Acta Cir Bras. [periódico na internet] 2006;21 Supl 3:8-15. Disponível em URL: http://www.scielo.br/acb

*Figuras coloridas disponíveis em $\underline{\text { www.scielo.br/acb }}$ 